TITLE:

\title{
Acute effect of multiple sets of fatiguing resistance exercise on muscle thickness, echo intensity and extracellular-to-intracellular water ratio
}

\section{$\operatorname{AUTHOR}(S)$ :}

Taniguchi, Masashi; Yamada, Yosuke; Ichihashi, Noriaki

\section{CITATION:}

Taniguchi, Masashi ...[et al]. Acute effect of multiple sets of fatiguing resistance exercise on muscle thickness, echo intensity and extracellular-to-intracellular water ratio. Applied Physiology, Nutrition, and Metabolism 2020, 45(2): 213-219

\section{ISSUE DATE:}

2020-02

URL:

http://hdl.handle.net/2433/261983

\section{RIGHT:}

This is the accepted manuscript of the article, which has been published at https://doi.org/10.1139/apnm-2018-0813.; この論文は出版社版でありません。引用の際 には出版社版をご確認ご利用ください。; This is not the published version. Please cite only the published version. 


\section{Title page}

Article title; Acute effect of multiple sets of fatiguing resistance exercise on muscle thickness, echo intensity and extracellular-to-intracellular water ratio.

The names of the authors; Taniguchi $\mathrm{M}^{1}$, Yamada $\mathrm{Y}^{2}$, and Ichihashi $\mathrm{N}^{1}$

The affiliations and addresses of the authors;

${ }^{1}$ Human Health Sciences, Graduate School of Medicine, Kyoto University; 53, Kawahara-cho, Shogoin, Sakyo-ku, Kyoto, 606-8507, Japan.

${ }^{2}$ National Institute of Health and Nutrition, National Institutes of Biomedical Innovation, Health and Nutrition; 1-23-1, Toyama, Shinjuku-ku, Tokyo, 162-8636, Japan.

Corresponding author contact information; Taniguchi M, RPT, PhD,

Email: taniguchi.masashi.7a@kyoto-u.ac.jpＴel.: +81-75-751-3964Ｆax:+81-: +81-75-751-3964

Contact information for other authors;

Yamada Y; yyamada831@gmail.com

Ichihashi N; ichihashi.noriaki.5z@kyoto-u.ac.jp 
Abstract: Resistance exercise (RE) causes an acute increase of the muscle thickness (MT) considered to relate to an increase in tissue water content. Segmental bioelectrical impedance spectroscopy (S-BIS) is a tool to non-invasively assess intra- and extra-cellular water (ICW and ECW, respectively) of a given limb segment. The purpose of the present study was to examine the change of MT, ultrasound echo intensity (EI), ICW and ECW after three sets of exhaustive RE. Eighteen untrained young males (age, $25.4 \pm 4.1$ years) performed RE consisting of three sets of knee extension concentric and eccentric contractions with $80 \%$ of one repetition maximum to failure. The MT and EI of the quadriceps measured by ultrasonography, and ECW/ICW ratio of the thigh assessed by S-BIS before (baseline) and after each set of RE (PostEx-1, 2, and 3). The changes $(\Delta)$ in MT, EI, and ECW/ICW ratio were calculated as values of PostEx minus baseline values. The values of MT, EI, and ECW/ICW ratio at PostEx-3 were significantly higher than baseline (effect size: MT, 1.11; EI, 0.47; and ECW/ICW ratio, 0.45). In addition, $\Delta \mathrm{MT}$ was significantly and moderately correlated with $\triangle \mathrm{ECW} / \mathrm{ICW}$ ratio $(\mathrm{r}=0.61)$. Integrated data showed weak but significant correlation between $\Delta \mathrm{EI}$ and $\triangle \mathrm{ECW} / \mathrm{ICW}$ ratio as well $(\mathrm{r}=0.31)$. The present results suggest multiple sets of exhaustive knee extension RE induce the acute increase of EI and ECW/ICW ratio as well as MT. - The acute increase of muscle size after exercise can be at least partly explained by relative ECW increase.

Keywords: resistance exercise; muscle thickness; echo intensity; extracellular-to-intracellular water ratio; extracellular fluid; edema; muscle swelling; muscle damage 


\section{Introduction}

Resistance exercise (RE) improves strength, and repetitive and adequately intense RE for weeks or months will induce skeletal muscle hypertrophy. It is known that RE causes an acute or delayed increase in muscle cross-sectional area (CSA) or muscle thickness (MT) (Damas et al. 2016; Yasuda et al. 2015). A previous study (Takahashi et al. 1994) measured the muscle CSA and T2 transverse relaxation time using magnetic resonance imaging (MRI). They both significantly increased immediately (0-7 min) after exercise, returned to baseline at 7-60 min, increased again at 12-48 $\mathrm{h}$, and returned to baseline again at seven days after knee extension eccentric exercise, i.e. bimodal responses with acute and delayed changes. The muscle CSA and T2 transverse relaxation time suggested that the acute change after exercise considered being involved an increase in total water content, particularly in extracellular water (ECW) fraction.

Segmental bioelectrical impedance spectroscopy (S-BIS) is a unique tool to estimate intracellular water (ICW) and ECW in a given limb segment without any radiation or invasion within seconds. Bioelectrical impedance analysis, including S-BIS, has been mainly used to evaluate body composition in a resting state in order to assess nutritional status of the subjects. However, S-BIS also has the potential to assess the change of ECW or ICW in a given limb, through estimation of these water contents before and after exercise (Shiose et al. 2018; Shiose et al. 2016). Changes in these water contents obtained by S-BIS have been associated with metabolic changes in skeletal muscle cells such as changes in intracellular $\mathrm{pH}$ and 
phosphocreatine levels during and after exercise (Raja et al. 2006). Since the relative increase in $\mathrm{ECW} / \mathrm{ICW}$ ratio, in particular the increase of $\mathrm{ECW}$, can indirectly reflect fluid shift to the extramyocellular space (Kagawa et al. 2014), this parameter may be associated with an acute increase of muscle size after RE.

Previous studies have reported that enhanced muscle echo intensity (EI) obtained by ultrasonography was concomitant with increased muscle size (Chen and Nosaka 2006; Gonzalez-Izal et al. 2014). The increase of EI is considered as an index of muscle tissue abnormality, muscle swelling, or muscle damage after the exercise (Fujikake et al. 2009; Rech et al. 2014). It is well known that eccentric contraction induces the delayed increase of EI at 2-5 days after exercise (Chen et al. 2012; Chen et al. 2010; Matta et al. 2018), but the acute change of EI inconsistent in previous literature (Jenkins et al. 2015; Muddle et al. 2019; Radaelli et al. 2012). A previous study conducted an elbow flexion exercise with 4 sets of 10 concentric-eccentric repetitions at $80 \%$ of 1 repetition maximum (RM) and found no significant change of EI immediately after exercise (Radaelli et al. 2012). In contrast, most recent study (Muddle et al. 2019) conducted knee extension exercise with repeated, fatiguing isometric contractions at $70 \%$ or $30 \%$ maximal isometric voluntary contraction (MIVC) and found significant increase of EI immediately after exercise. The inconsistency found in the findings of these studies could be due to differences in training volume and the muscle groups. Exhaustive RE with large training volume could cause an immediate EI increase even in large muscles. Therefore, we hypothesize that the multiple sets of exhaustive RE until 
repetition failure with large muscles is needed to obtain significant and acute increase of EI.

The purpose of the present study was to examine the acute changes of MT, EI and ECW/ICW ratio during and after three sets of fatiguing knee extension RE. Our hypotheses are 1) MT, EI, and ECW/ICW ratio increase after the final RE set, and not after the first RE set, and 2) the acute increase of MT is correlated with the expansion of ECW/ICW ratio.

\section{Materials and methods}

\section{Participants}

Eighteen untrained young males (mean age, $25.4 \pm 4.1$ years; height, $170.3 \pm 5.9 \mathrm{~cm}$; body weight, $62.3 \pm$ $8.9 \mathrm{~kg}$ ) were recruited in the present study. The inclusion criteria were no routine lower-limb RE and no intake of any supplements or anti-inflammatory medications for at least six months. The sample size was determined based on our preliminary data that investigated the MT on the quadriceps between pre- and post-RE until fatigue failure. Based on the effect size (ES) of 0.8 in MT between pre- and post-RE, alpha-level of 0.05 , and a beta level of 0.20 , it was calculated that at least 15 participants were necessary. Considering the possibility of missing data and measurement variation, our study recruited 18 participants. The sensitivity (r) for correlation analysis was 0.58 based on a sample size of 18 , an alpha level of 0.05 , and a beta level of 0.20 (i.e., 18 participants were sufficient to detect a true effect of $r=$ 
0.6). All study protocols were approved by the ethics committee of Kyoto University. All subjects received adequate explanation of the study protocols and provided written consent.

\section{Experimental procedures}

All participants underwent a knee extension 1RM test more than three days before the exhaustive RE protocol. All participants were instructed to keep their lifestyle and avoid high-intensity physical activity from the time of the knee extension 1RM test to the main experiment. Additionally, the investigator confirmed that the participants had no muscle soreness or pain at the time of the main experiment. Figure 1 shows the time course of the RE sessions, rest intervals, and measurements. The measurements of ultrasound images and S-BIS were performed before (baseline) and after each set of RE (PostEx-1, 2, and 3). These measurements were performed within 3 min after each RE.

\section{RM testing protocols}

All participants tested unilateral 10RM strength for their right leg in a variable resistance knee extension machine (FX-50, Paramount Fitness, USA). The participants were seated straight on the machine, with their feet off the floor. In estimating the knee extension 1RM, the 10RM load conversion guidelines of the National Strength and Conditioning Association were used (Baechle and Earle 2010). The participants were familiarised with knee extension loads with a light weight of their choice before attempting 10RM. 
If participant completed the 10RM testing, the investigator chose the weight increase for each attempt,

and adequate rest time (approximately $3 \mathrm{~min}$ ) between attempts was provided. In cases where the final load was over- or underestimated, the participants performed a modified attempt with a lower or higher load. The knee extension 1RM strength was obtained within 3-5 attempts (Barcelos et al. 2015).

\section{The knee extension exercise until fatigue failure}

RE on the quadriceps was performed using a knee extension machine (FX-50, Paramount Fitness, St.

Louis, USA). The load for each participant was set to an intensity of approximately $80 \% 1 \mathrm{RM}$. After understanding the exercise protocol, the knee extension exercise was performed between $90^{\circ}$ to $0^{\circ}\left(0^{\circ}=\right.$ full knee extension) in the concentric and eccentric phases until fatigue failure. Additionally, the participants were instructed to exercise in accordance with the unified cadence of 50 beats per min set by the metronome (Burd et al. 2010). The investigator gave verbal encouragement and cautioned to maintain the exercise protocols, especially the motion cadence in the eccentric phase. Fatigue failure was defined as a condition of unsuccessfully maintaining the motion cadence and knee extension angle of $45^{\circ}$ or less. The participants performed three sets of fatigue failure with $80 \%$ 1RM load and had a 3-min rest interval between each set. Total exercise volume was evaluated as load $(\mathrm{kg}) \times$ repetitions $\times$ sets.

\section{The evaluation of subjective fatigue and strength}


Subjective fatigue on the right exercise limb was measured using a Borg scale pre- and post-RE.

Moreover, knee extension maximum strength was tested before and after RE to evaluate the degree of muscle fatigue. The participants were positioned on the dynamometer (Isoforce GT-330; OG Giken, Okayama, Japan) with their knee flexed at $60^{\circ}$ and were instructed to generate a MIVC for $3 \mathrm{~s}$. The MIVC was measured for three attempts, and the highest value was accepted. In addition, muscle fatigue index was calculated as the percentage reduction in knee extension maximum strength between pre- and post-RE.

\section{Ultrasound images}

Prior to the RE session, the participants had a rest time during which they relaxed in supine position for 15-20 min. The rest time prior to ultrasound measurement was set according to the BIS measurement. A B-mode ultrasound device equipped with an 8-MHz linear probe was used to obtain transverse ultrasound images (F37; Hitachi Aloka Medical, Tokyo, Japan). Equipment settings included dynamic range of 69 $\mathrm{dB}$, gain of $58 \mathrm{~dB}$, and time gain compensation in the neutral position, and these settings were maintained, with the same examiner performing all measurements. In accordance with a previous study (Taniguchi et al. 2015), measurement sites of each quadriceps muscle were determined, and the MT of rectus femoris, vastus intermedius, vastus lateralis, and vastus medialis was measured. The MT of the whole quadriceps (Sum-Quad MT) was defined as the summed MT of the rectus femoris, vastus 
intermedius, vastus lateralis, and vastus medialis. An image processing software (ImageJ-WinJP, LISIT, Tokyo, Japan) was used to analyse the EI, and the average EI of the rectus femoris, vastus lateralis, and vastus medialis muscles was calculated. As the EI values depend on the depth of the muscle of interest, the vastus intermedius was excluded from the analysis (Fukumoto et al. 2012). A previous study reported that the MT and EI of the whole quadriceps group were strongly correlated with muscle strength (Wilhelm et al. 2014); therefore, the summed MT of all four quadriceps muscle heads and averaged EI of three heads, excluding the vastus intermedius, was used in the present study. The within-day coefficients of variation $(\mathrm{CV})$ for repeated MT and EI measures in our laboratory were $0.8 \%$ and $3.9 \%$, and standard error mean (SEM) were 0.074 and 0.183 . The between-day CV for repeated MT and EI measures were $1.2 \%$ and $4.6 \%$, and SEM were 0.106 and 0.298 .

\section{Segmental bioelectrical impedance spectroscopy}

The S-BIS measurements were conducted $15-20 \mathrm{~min}$ after the participants remained in the supine position and after measuring ultrasound images to consider the immediate shift of body fluids (Kushner et al. 1996). According to a previous study (Taniguchi et al. 2017), the body impedance values were obtained from the thigh in the right side using a BIS device (MLT550; SK Medical, Shiga, Japan), which had a logarithmic spectrum of 140 frequencies. The disposable electrodes $(42.5 \times 38 \mathrm{~mm}$, Blue Sensor SP-00-S; Mets Inc., Tokyo, Japan) were placed on the following four sites: dorsal surfaces of the hand 
and foot, anterior superior iliac spine, and lateral knee articular condyles. The distance between the anterior superior iliac spine and lateral knee articular condyles was measured as segmental length. S-BIS measurements were performed for three consecutive repetitions to obtain bioelectrical impedance on the upper thigh. Acquisition, storage, and analysis of data were performed using the BIS equipment software. The results of S-BIS were provided as follows: the resistance (R) of the ECW compartment $\left(\mathrm{R}_{\mathrm{ECW}}=\mathrm{R}_{0}\right)$ and ICW compartment $\left(\mathrm{R}_{\mathrm{ICW}}=1 /\left[1 / \mathrm{R}_{\infty}-1 / \mathrm{R}_{0}\right]\right)$ was identified by fitting the spectrum of bioimpedance value to the Cole-Cole model. The calculation of the ICW and ECW was performed with the algorithm in accordance with previous studies (Kaysen et al. 2005; Yamada et al. 2013; Zhu et al. 1998). The algorithm was that $\mathrm{ECW}=\rho \mathrm{ECW} \times(\text { segmental length })^{2} / \mathrm{R}_{\infty}$ and $\mathrm{ICW}=\rho \mathrm{ICW} \times(\text { segmental length })^{2} /$ $\mathrm{R}_{\mathrm{ICW}} \rho$ represents the specific resistivity, $\rho \mathrm{ECW}=47 \Omega \mathrm{m}$, and $\rho \mathrm{ICW}=273.9 \Omega \mathrm{m}$. The mean ECW and ICW values in the three repetitions were used for subsequent analysis. The estimation of ECW and ICW was associated with segmental volume; thus, conversion to the ECW/ICW could indicate a percentage of water content in the extramyocellular space regardless of the somatotype. The bioelectrical impedance value is affected by the shift in body fluid on postural transition (Kagawa et al. 2014). In the present study, to confirm the influence of S-BIS measurement on postural transition, the change in ECW/ICW ratio was investigated in a relaxed supine position for approximately $30 \mathrm{~min}$, which corresponded to the time required for all experimental protocols. As a result, the ECW/ICW ratio had a small change in time course (participants, five young men; before, $32.9 \pm 4.1 \%$, after $30 \mathrm{~min}, 32.8 \pm 4.2 \% ; \Delta, 0.14 \pm 0.19 \%$ ). 
The within- and between-day $\mathrm{CV}$ for repeated $\mathrm{ECW} / \mathrm{ICW}$ ratio measures in our laboratory was $2.6 \%$ and

$2.8 \%$, and SEM was 0.245 and 0.607 , respectively.

\section{Statistical analysis}

The Sum-Quad MT, EI, and ECW/ICW were measured before (baseline) and after each set of RE

(PostEx-1, 2, and 3), and changes $(\Delta)$ in these variables were calculated as values of PostEx minus baseline values. All values are presented as mean \pm standard deviation (SD). The statistical procedure was performed using SPSS software version 22.0 (SPSS Japan Inc., Tokyo, Japan). Paired-t test was used for differences in muscle properties and fatigue parameters between baseline and PostEx3, and the ES of muscle properties between baseline and PostEx3 was calculated with Cohen's d. In a secondary analysis, one-way analysis of variance (ANOVA) and post hoc test with the Tukey HSD adjustment were performed to test for the time course of muscle property changes between RE sets. Additionally, tests for linear trends were conducted on these parameters between RE sets. The level of significance was set at $p$ $<0.05$. The relationship between $\Delta$ Sum-Quad MT, $\Delta \mathrm{EI}$, and $\Delta \mathrm{ECW} / \mathrm{ICW}$ ratio were obtained as two ways; using only data after three sets of RE $(n=18)$, and using all points of data at PostEx1, 2, and $3(n=$ 54 in total). With a sample size of 54 , the sensitivity (r) for the correlation analysis was calculated as 0.36 based on an alpha level of 0.05 and a beta level of 0.20 . Although a sample size of 18 is statistically significant with moderate correlation coefficients $(\mathrm{r}=0.58, \mathrm{p}<0.05$, and power of 0.80$)$, a sample size of 
54 seems to have a weak correlation $(\mathrm{r}=0.36)$. Statistical comparison between correlation coefficients was performed using Meng's z-test (Meng XL et al. 1992).

\section{Results}

Participants performed RE sets until fatigue as follows: mean $1 \mathrm{RM}=62.8 \pm 8.3 \mathrm{~kg}$, total exercise repetitions $=36.2 \pm 4.6(\mathrm{RE} 1=13.9 \pm 2.1, \mathrm{RE} 2=12.1 \pm 1.9$, and RE3 $=10.2 \pm 1.5)$, and total exercise volume $=2974 \pm 673 \mathrm{~kg}$. MIVC was significantly smaller in PostEx3 compared to baseline (baseline, $171.1 \pm 37.2 \mathrm{Nm}$; PostEx3, $134.4 \pm 34.4 \mathrm{Nm} ; \mathrm{p}<0.001$; muscle fatigue index, $-21.3 \pm 11.1 \%)$, and Borg scale values were significantly larger (baseline, median 6 points; PostEx3, 16 points; 95\% confidence interval (CI) 8.7 to $10.7, \mathrm{p}<0.001)$.

The values of muscle properties at baseline and PostEx3 are presented in Table 1. Sum-Quad MT, EI, and ECW/ICW ratio significantly increased at PostEx3 compared with the baseline values. As supplemental information on ECW/ICW ratio change, ECW significantly increased at Post Ex3 compared with the baseline value, whereas the ICW value had no significant change between baseline and PostEx3. The change $(\Delta)$ and ES were also shown in Table 1.

Figure 2 shows the time course changes in Sum-Quad MT, EI, and ECW/ICW ratio over RE sets. One-way ANOVA showed a significant set difference in $\triangle$ Sum-Quad MT $(F=15.62, p<0.001)$, 
$\Delta \mathrm{EI}(\mathrm{F}=6.31, \mathrm{p}=0.004)$, and $\Delta \mathrm{ECW} / \mathrm{ICW}$ ratio $(\mathrm{F}=7.59, \mathrm{p}=0.001) . \Delta$ Sum-Quad $\mathrm{MT}$ increased significantly across all measurement periods, while $\Delta \mathrm{EI}$ and $\triangle \mathrm{ECW} / \mathrm{ICW}$ ratio increased significantly only in PostEx3 with respect to PostEx1 and PostEx2. The time course trends of $\Delta \mathrm{EI}$ and $\Delta \mathrm{ECW} / \mathrm{ICW}$ ratio were similar $(\mathrm{P}$ for trend $<0.01)$.

Figure 3 shows the scatter plots between $\triangle \mathrm{Sum}$-Quad MT, $\triangle \mathrm{ECW} / \mathrm{ICW}$ ratio, and $\triangle \mathrm{EI}$ after three sets of RE. $\triangle$ Sum-Quad MT significantly and positively correlated with $\triangle E C W /$ ICW ratio $(r=0.61$, $95 \% \mathrm{CI} ; 0.20$ to $0.84, \mathrm{p}<0.01)$, but not with $\Delta \mathrm{EI}(\mathrm{r}=-0.26,95 \% \mathrm{CI} ;-0.65$ to $0.24, \mathrm{p}=0.31)$. Additionally, there was no significant correlation between $\Delta \mathrm{EI}$ and $\Delta \mathrm{ECW} / \mathrm{ICW}$ ratio $(\mathrm{r}=-0.16,95 \% \mathrm{CI} ;-0.58$ to 0.33 , $\mathrm{p}=0.52)$. As the result of Meng's z-test, the correlation coefficients between $\Delta$ Sum-Quad MT and $\triangle \mathrm{ECW} / \mathrm{ICW}$ ratio was significantly larger than that between $\Delta$ Sum-Quad MT and $\Delta \mathrm{EI}(\mathrm{p}=0.01)$. Figure 4 shows the scatter plots between $\Delta$ Sum-Quad MT, $\triangle \mathrm{ECW} / \mathrm{ICW}$ ratio, and $\Delta \mathrm{EI}$ after three sets of RE with all of time course datasets (PostEx1, 2, and 3). The correlation between $\Delta$ Sum-Quad MT and $\triangle \mathrm{ECW} / \mathrm{ICW}$ ratio was still significant $(\mathrm{p}<0.01)$. The $\Delta \mathrm{EI}$ was weakly but significantly correlated with $\Delta \mathrm{ECW} / \mathrm{ICW}$ ratio $(\mathrm{p}<0.05)$ in the merged dataset $(\mathrm{n}=54)$.

\section{Discussion}

In the present study, the MT increase gradually after each set of exhaustive RE. In contrast, the values of 
EI and ECW/ICW ratio at PostEx3, but not at PostEx1 or 2, were significantly higher than those at baseline. Additionally, a significant and positive correlation between $\triangle \mathrm{Sum}$-Quad MT and $\triangle \mathrm{ECW} / \mathrm{ICW}$ ratio was observed. EI significantly increased after three RE sets, whereas the correlation between $\Delta$ Sum-Quad MT and $\Delta$ EI was not significant. These results have suggested that the acute expansion of muscle size after multiple sets of exhaustive RE was at least partly explained by the relative expansion of ECW.

While the increase of post-RE ECW/ICW ratio was relevant to our hypothesis, we additionally reported ECW and ICW independently to explain the ECW/ICW ratio changes. Compared to baseline, PostEx3 ECW and ICW were significantly greater and not significantly different, therefore implying that ECW/ICW ratio increases were primarily due to increases of ECW. Previous studies (Sjogaard and Saltin 1982; Sjogaard et al. 1985) indicated that total water (TW) and ECW, measured by radio-isotope [3H]-inulin dilution space, of the vastus lateralis muscle tissue obtained by biopsy significantly increased after three sets of bicycle ergometer exercise with 3-min 120\% maximal oxygen uptake, with non-significant and small change of ICW. Thus, the fluid volume in muscle tissues, including extracellular fractions between muscle fibers and muscle bundles, will change after exhaustive RE. Another study (Raja et al. 2006) showed that ICW increased during RE and immediately returned to pre-RE levels during recovery phase, whereas ECW volume tended to increase $\sim 8-9 \%$ during $8-12$ minutes post-RE. The muscle cell volume and ICW is well regulated by complex mechanisms, and the 
muscle cell swelling induced by muscle fiber contractions returns quickly (Usher-Smith et al. 2009). For that reason, the present study, as well as the previous studies (Sjogaard and Saltin 1982; Sjogaard et al. 1985), could not find any significant change of ICW after exercise. A careful time course study is needed to observe ICW change. For ECW (interstitial plus plasma fluid), potential mechanisms of the expansion of ECW are the water shift from muscle cells to interstitial space, exercise hyperaemia and increased blood flow. The time course change in MT was a little bit different from that of EI or ECW/ICW ratio during three sets of exhaustive RE. Although $\Delta$ Sum-Quad MT showed significant increased step by step between RE sets, $\triangle \mathrm{ECW} / \mathrm{ICW}$ ratio showed a significant increase in PostEx3 compared with those in PostEx1 and PostEx2. Since previous study (Raja et al. 2006) has shown that the increase of ECW occurred a while after stopping RE, the change in ECW/ICW ratio could have a time delay. Thus fluid volume change time courses need to be considered both during and after RE sets.

Since EI can be easily obtained with other muscle morphologies using ultrasonography, many studies assessed EI with resting state or exercise (Jenkins et al. 2015; Muddle et al. 2019; Radaelli et al. 2012). However, it is not clear what exactly the increase of EI after exercise means (Fujikake et al. 2009). In resting state, higher value of EI is related to higher proportion of intramuscular adipose, fibrous, or connective tissues (Pillen et al. 2009; Watanabe et al. 2018). In animal model, focal edema and inflammation fibers were observed several days after eccentric exercise (Sudo and Kano 2009). The EI is also increased several days after exercise in animal (Rech et al. 2014) and human (Chen et al. 2012; Chen 
et al. 2010). A previous study (Fujikake et al. 2009) using rat tibialis anterior muscles observed EI change after injection of bupivacaine hydrochloride (BPVC) that induced muscle damage. The EI peaks were observed immediately after injection of BPVC and the peak values are same with saline injection. In contrast, the time course change of EI was different, and the EI value returned to baseline $24 \mathrm{~h}$ after saline injection, but the EI value still significantly higher even 14 days after BPVC injection. They stated "time course change of EI did not correspond to the infiltration of inflammatory cells shown in the histologic analysis such that the number of cells increased significantly after $24 \mathrm{~h}$ post BPVC injection when the echo intensity decreased from its peak value" (Fujikake et al. 2009). In that study, both condition of BPVC and saline injections induced an enlargement of interstitial space and swelling fibers. They interpreted that increase in interstitial fluid, i.e. edema, will induce increase in EI after muscle damage, with an assumption of a large difference of acoustic impedances between ECW and muscle cells. The time course trends of $\triangle \mathrm{EI}$ and $\triangle \mathrm{ECW} / \mathrm{ICW}$ ratio were similar, and weak but significant correlation between $\triangle \mathrm{EI}$ and $\triangle \mathrm{ECW} / \mathrm{ICW}$ ratio was observed in the merged dataset in the present study. These results at least partly support the previous interpretation. Therefore, although both $\Delta \mathrm{EI}$ and $\triangle \mathrm{ECW} / \mathrm{ICW}$ ratios could explain the exercise-induced muscle edema, change in EI may reflect the delayed effect rather than the acute change after RE. However, we did not observed delayed effect of muscle damage several days after exercise and the EI have larger measurement error than other variables potentially. The observed correlation was small in the present study. In addition, the acute change of EI after exercise is inconsistent 
between literature (Jenkins et al. 2015; Muddle et al. 2019; Radaelli et al. 2012). Thus, our results on the acute change in EI should not be overinterpreted, and further studies on what change in EI after exercise actually means are needed.

This study has some limitations. Firstly, the present study only examined the changes on MT, EI and $\mathrm{ECW} / \mathrm{ICW}$ ratio immediately after each set of RE (acute change), and did not observed long-term changes, such as over minutes, hours, and days or weeks after exercise. The time course in the recovery phase could be different. Further studies are needed to pay attention to such time courses changes. Secondly, this study did not measure movement velocity during RE. The changes in movement velocity could have effected concentric (CON) and eccentric (ECC) fatigue. Physiologically, CON and ECC contractions affect differently to muscle properties after exercise. Thus, it may have been preferable to perform RE using a dynamometer that can control the movement velocity and the contraction phase or perform ECC and CON exercises separately. Thirdly, a previous study has suggested that ECC exercise-induced muscle damage assessed from EI was different among elbow and knee flexors or extensors (Chen et al. 2011), and knee extension was less sensitive to observe delayed muscle damage than other exercise modality. In addition, we did not measure any other biomarkers related to muscle damage or muscle swelling. Replication studies are needed to verify our results using different muscle groups to generalize in our result, and further studies are needed to understand physiological mechanisms of the changes in water distribution in muscle tissue with various biomarkers. We also acknowledge that 
measured impedance is related not only to fluid volume but also to its intrinsic resistivity. Therefore, S-BIS is potentially influenced by ionic concentrations in the aqueous space. The S-BIS is non-radiation, non-invasion, portable, and easy-to-use devise, but still needs some assumptions to estimate water distribution.

\section{Conclusion}

The present results suggest multiple sets of exhaustive knee extension RE induce the acute increase of EI and $\mathrm{ECW} / \mathrm{ICW}$ ratio as well as MT. $\triangle \mathrm{MT}$ was significantly and moderately correlated with $\triangle \mathrm{ECW} / \mathrm{ICW}$ ratio, whereas correlation between $\Delta \mathrm{MT}$ and $\Delta \mathrm{EI}$ was not significant. Therefore, the immediate increase of MT after three-sets of exhaustive RE can be partly explained by relative expansion of ECW against ICW. The present study suggested that S-BIS may be a useful tool to evaluate the status of the acute response associated with post-RE muscle size increases. 


\section{Acknowledgments}

The authors would like to thank Dr. Nosaka K (Edith Cowan University) for his insightful advice in the deep discussion. The authors also thank all of the individuals who participated in the study. This study was not funded by any institutions, agencies, or companies.

\section{Conflicts of interest}

The authors have no conflicts of interest to disclose.

\section{References}

Baechle, T.R., and Earle, R.W. 2010. Essentials of strength training and conditioning Japanese translation. Book House HD.

Barcelos, L.C., Nunes, P.R., de Souza, L.R., de Oliveira, A.A., Furlanetto, R., Marocolo, M., et al. 2015. Low-load resistance training promotes muscular adaptation regardless of vascular occlusion, load, or volume. Eur J Appl Physiol 115(7): 1559-1568. doi:10.1007/s00421-015-3141-9.

Burd, N.A., West, D.W., Staples, A.W., Atherton, P.J., Baker, J.M., Moore, D.R., et al. 2010. 
Low-load high volume resistance exercise stimulates muscle protein synthesis more than

high-load low volume resistance exercise in young men. PLoS One 5(8): e12033. doi:10.1371/journal.pone.0012033.

Chen, H.L., Nosaka, K., Pearce, A.J., and Chen, T.C. 2012. Two maximal isometric contractions attenuate the magnitude of eccentric exercise-induced muscle damage. Applied physiology, nutrition, and metabolism = Physiologie appliquee, nutrition et metabolisme 37(4): 680-689. doi:10.1139/h2012-035.

Chen, T.C., and Nosaka, K. 2006. Responses of elbow flexors to two strenuous eccentric exercise bouts separated by three days. J Strength Cond Res 20(1): 108-116. doi:10.1519/R-16634.1.

Chen, T.C., Chen, H.L., Lin, M.J., Wu, C.J., and Nosaka, K. 2010. Potent protective effect conferred by four bouts of low-intensity eccentric exercise. Med Sci Sports Exerc 42(5): 1004-1012. doi:10.1249/MSS.0b013e3181c0a818.

Damas, F., Phillips, S.M., Lixandrao, M.E., Vechin, F.C., Libardi, C.A., Roschel, H., et al. 2016. Early resistance training-induced increases in muscle cross-sectional area are concomitant with edema-induced muscle swelling. Eur J Appl Physiol 116(1): 49-56. doi:10.1007/s00421-015-3243-4.

Fujikake, T., Hart, R., and Nosaka, K. 2009. Changes in B-mode ultrasound echo intensity 
following injection of bupivacaine hydrochloride to rat hind limb muscles in relation to

histologic changes. Ultrasound Med Biol 35(4): 687-696.

doi:10.1016/j.ultrasmedbio.2008.10.008.

Fukumoto, Y., Ikezoe, T., Yamada, Y., Tsukagoshi, R., Nakamura, M., Mori, N., et al. 2012.

Skeletal muscle quality assessed from echo intensity is associated with muscle strength

of middle-aged and elderly persons. Eur J Appl Physiol 112(4): 1519-1525.

doi:10.1007/s00421-011-2099-5.

Gonzalez-Izal, M., Lusa Cadore, E., and Izquierdo, M. 2014. Muscle conduction velocity,

surface electromyography variables, and echo intensity during concentric and eccentric

fatigue. Muscle Nerve 49(3): 389-397. Available from

https://www.ncbi.nlm.nih.gov/pubmed/24741684.

Jenkins, N.D., Housh, T.J., Bergstrom, H.C., Cochrane, K.C., Hill, E.C., Smith, C.M., et al.

2015. Muscle activation during three sets to failure at 80 vs. 30\% 1RM resistance exercise.

Eur J Appl Physiol 115(11): 2335-2347. doi:10.1007/s00421-015-3214-9.

Kagawa, M., Wishart, C., and Hills, A.P. 2014. Influence of posture and frequency modes in total body water estimation using bioelectrical impedance spectroscopy in boys and adult males. Nutrients 6(5): 1886-1898. doi:10.3390/nu6051886.

Kaysen, G.A., Zhu, F., Sarkar, S., Heymsfield, S.B., Wong, J., Kaitwatcharachai, C., et al. 
2005. Estimation of total-body and limb muscle mass in hemodialysis patients by using

multifrequency bioimpedance spectroscopy. Am J Clin Nutr 82(5): 988-995. Available from http://www.ncbi.nlm.nih.gov/pubmed/16280429.

Kushner, R.F., Gudivaka, R., and Schoeller, D.A. 1996. Clinical characteristics influencing bioelectrical impedance analysis measurements. Am J Clin Nutr 64(3 Suppl): 423S-427S. Available from http://www.ncbi.nlm.nih.gov/pubmed/8780358.

Matta, T.T.D., Pereira, W.C.A., Radaelli, R., Pinto, R.S., and Oliveira, L.F. 2018. Texture analysis of ultrasound images is a sensitive method to follow-up muscle damage induced by eccentric exercise. Clin Physiol Funct Imaging 38(3): 477-482. doi:10.1111/cpf.12441.

Meng XL, Rosenthal R, and DB., R. 1992. Comparing correlated correlation-coefficients. Psychol Bull 111: 172-175.

Muddle, T.W.D., Magrini, M.A., Colquhoun, R.J., Luera, M.J., Tomko, P.M., and Jenkins, N.D.M. 2019. Impact of Fatiguing, Submaximal High- vs. Low-Torque Isometric Exercise on Acute Muscle Swelling, and Echo Intensity in Resistance-Trained Men. J Strength Cond Res 33(4): 1007-1019. doi:10.1519/JSC.0000000000003033.

Pillen, S., Tak, R.O., Zwarts, M.J., Lammens, M.M., Verrijp, K.N., Arts, I.M., et al. 2009. Skeletal muscle ultrasound: correlation between fibrous tissue and echo intensity. Ultrasound Med Biol 35(3): 443-446. doi:10.1016/j.ultrasmedbio.2008.09.016. 
Radaelli, R., Bottaro, M., Wilhelm, E.N., Wagner, D.R., and Pinto, R.S. 2012. Time course of strength and echo intensity recovery after resistance exercise in women. J Strength Cond Res 26(9): 2577-2584. doi:10.1519/JSC.0b013e31823dae96.

Raja, M.K., Raymer, G.H., Moran, G.R., Marsh, G., and Thompson, R.T. 2006. Changes in tissue water content measured with multiple-frequency bioimpedance and metabolism measured with 31P-MRS during progressive forearm exercise. J Appl Physiol (1985) 101(4): 1070-1075. doi:10.1152/japplphysiol.01322.2005.

Rech, A., Radaelli, R., De Assis, A.M., Fernandes, J.R., Longoni, A., Vozari-Hampe, M.M., et al. 2014. The effects of strength, aerobic, and concurrent exercise on skeletal muscle damage in rats. Muscle Nerve 50(1): 79-86. doi:10.1002/mus.24091.

Shiose, K., Yamada, Y., Motonaga, K., and Takahashi, H. 2018. Muscle glycogen depletion does not alter segmental extracellular and intracellular water distribution measured using bioimpedance spectroscopy. J Appl Physiol (1985) 124(6): 1420-1425. doi:10.1152/japplphysiol.00666.2017.

Shiose, K., Yamada, Y., Motonaga, K., Sagayama, H., Higaki, Y., Tanaka, H., et al. 2016. Segmental extracellular and intracellular water distribution and muscle glycogen after 72-h carbohydrate loading using spectroscopic techniques. J Appl Physiol (1985) 121(1): 205-211. doi:10.1152/japplphysiol.00126.2016. 
Sjogaard, G., and Saltin, B. 1982. Extra- and intracellular water spaces in muscles of man at rest and with dynamic exercise. Am J Physiol 243(3): R271-280. doi:10.1152/ajpregu.1982.243.3.R271.

Sjogaard, G., Adams, R.P., and Saltin, B. 1985. Water and ion shifts in skeletal muscle of humans with intense dynamic knee extension. Am J Physiol 248(2 Pt 2): R190-196. doi:10.1152/ajpregu.1985.248.2.R190.

Sudo, M., and Kano, Y. 2009. Myofiber apoptosis occurs in the inflammation and regeneration phase following eccentric contractions in rats. J Physiol Sci 59(6): 405-412. doi:10.1007/s12576-009-0049-3.

Takahashi, H., Kuno, S., Miyamoto, T., Yoshioka, H., Inaki, M., Akima, H., et al. 1994. Changes in magnetic resonance images in human skeletal muscle after eccentric exercise.

Eur J Appl Physiol Occup Physiol 69(5): 408-413. Available from https://www.ncbi.nlm.nih.gov/pubmed/7875137.

Taniguchi, M., Fukumoto, Y., Kobayashi, M., Kawasaki, T., Maegawa, S., Ibuki, S., et al. 2015. Quantity and Quality of the Lower Extremity Muscles in Women with Knee $\begin{array}{llll}\text { Osteoarthritis. } & \text { Ultrasound } & \text { Med } & \text { Biol }\end{array}$ doi:10.1016/j.ultrasmedbio.2015.05.014.

Taniguchi, M., Yamada, Y., Fukumoto, Y., Sawano, S., Minami, S., Ikezoe, T., et al. 2017. 
Increase in echo intensity and extracellular-to-intracellular water ratio is independently associated with muscle weakness in elderly women. Eur J Appl Physiol 117(10): 2001-2007. doi:10.1007/s00421-017-3686-x.

Usher-Smith, J.A., Huang, C.L., and Fraser, J.A. 2009. Control of cell volume in skeletal muscle. Biol Rev Camb Philos Soc 84(1): 143-159. doi:10.1111/j.1469-185X.2008.00066.x.

Watanabe, Y., Ikenaga, M., Yoshimura, E., Yamada, Y., and Kimura, M. 2018. Association between echo intensity and attenuation of skeletal muscle in young and older adults: a comparison between ultrasonography and computed tomography. Clin Interv Aging 13: 1871-1878. doi:10.2147/CIA.S173372.

Wilhelm, E.N., Rech, A., Minozzo, F., Radaelli, R., Botton, C.E., and Pinto, R.S. 2014. Relationship between quadriceps femoris echo intensity, muscle power, and functional capacity of older men. Age 36(3): 9625. doi:10.1007/s11357-014-9625-4.

Yamada, Y., Watanabe, Y., Ikenaga, M., Yokoyama, K., Yoshida, T., Morimoto, T., et al. 2013. Comparison of single- or multifrequency bioelectrical impedance analysis and spectroscopy for assessment of appendicular skeletal muscle in the elderly. J Appl Physiol (1985) 115(6): 812-818. doi:10.1152/japplphysiol.00010.2013.

Yasuda, T., Fukumura, K., Iida, H., and Nakajima, T. 2015. Effect of low-load resistance exercise with and without blood flow restriction to volitional fatigue on muscle swelling. 
Eur J Appl Physiol 115(5): 919-926. doi:10.1007/s00421-014-3073-9.

Zhu, F., Schneditz, D., Wang, E., and Levin, N.W. 1998. Dynamics of segmental extracellular volumes during changes in body position by bioimpedance analysis. J Appl $\begin{array}{lllll}\text { Physiol } & \text { (1985) } & \text { 85(2): } & 497-504 . & \text { Available }\end{array}$ http://www.ncbi.nlm.nih.gov/pubmed/9688726. 
Table 1 The values of muscle thickness, echo intensity, and water fluid before (baseline) and after three sets of knee extension exercise to repetition failure (PostEx-3).

\begin{tabular}{|c|c|c|c|c|c|c|}
\hline & baseline & PostEx 3 & Change $(\Delta)$ & p-value & $95 \% \mathrm{CI}$ & ES \\
\hline Sum-Quad MT (cm) & $11.3 \pm 1.8$ & $13.3 \pm 1.8$ & $2.0 \pm 0.5$ & $<0.001$ & $1.69-2.24$ & 1.11 \\
\hline EI (A.U) & $16.1 \pm 5.3$ & $18.6 \pm 5.3$ & $2.5 \pm 2.0$ & $<0.001$ & $1.49-3.47$ & 0.47 \\
\hline $\mathrm{ECW} / \mathrm{ICW}$ ratio (\%) & $32.6 \pm 6.3$ & $35.4 \pm 6.9$ & $2.9 \pm 2.7$ & $<0.001$ & $1.49-4.21$ & 0.45 \\
\hline ICW (ml) & $3647 \pm 1094$ & $3555 \pm 1094$ & $-92 \pm 311$ & 0.227 & $-62.6-246.2$ & 0.08 \\
\hline ECW (ml) & $1141 \pm 233$ & $1216 \pm 233$ & $75 \pm 27$ & $<0.001$ & $-88.3--61.4$ & 0.32 \\
\hline
\end{tabular}

Note: MT: Muscle Thickness, EI: Echo Intensity, ECW/ICW ratio: Intracellular water / Extracellular water ratio, ICW: Intracellular water, ECW: Extracellular water, CI: Confidence Interval, ES: Effect Size. 


\section{Figure captions}

Figure 1. Time course of the resistance exercise session and measurement periods. After participants visited the laboratory, they relaxed in a supine position for 15-20 min to allow for the immediate shift of body fluids. The participants then performed RE consisting of three sets of knee extensions with $80 \%$ 1RM to failure. The Sum-Quad MT, EI, and ECW/ICW ratio were measured before (baseline) and after each set of RE (PostEx-1, 2, and 3) with resting position. Note: MIVC; maximal isometric voluntary contraction

Figure 2. Time course changes of (A) Sum-Quad muscle thickness (MT), (B) echo intensity (EI), and (C) extracellular-to-intracellular water ratio $(\mathrm{ECW} / \mathrm{ICW})$ over the three sets of RE. The values were measured before (baseline) and after each set of Ex (PostEx-1, 2, and 3). The changes $(\Delta)$ of these variables were calculated as values of PostEx minus baseline values. $*, p<0.05 ; * *, p<0.01$.

Figure 3. Scatter plots the changes of Sum-Quad muscle thickness (MT), echo intensity (EI), and extracellular-to-intracellular water ratio $(\mathrm{ECW} / \mathrm{ICW})$ after three sets of RE $(\mathrm{n}=18)$. The changes $(\Delta)$ of these variables were calculated as values of PostEx-3 minus baseline values. Panel A shows the correlation between $\Delta$ Sum-Quad MT and $\Delta \mathrm{EI}, \mathrm{B}$ shows the correlation between $\Delta \mathrm{ECW} / \mathrm{ICW}$ ratio and $\Delta \mathrm{EI}$, and $\mathrm{C}$ shows the correlation between $\Delta \mathrm{Sum}-\mathrm{Quad} \mathrm{MT}$ and $\triangle \mathrm{ECW} / \mathrm{ICW}$ ratio. 
Figure 4. Scatter plots the changes of Sum-Quad muscle thickness (MT), echo intensity (EI), and extracellular-to-intracellular water ratio $(\mathrm{ECW} / \mathrm{ICW})$ with all of time course datasets $(\mathrm{n}=54)$. The changes $(\Delta)$ of these variables were calculated as values of PostEx minus baseline values. Panel A shows the correlation between $\Delta$ Sum-Quad MT and $\Delta \mathrm{EI}, \mathrm{B}$ shows the correlation between $\Delta \mathrm{ECW} / \mathrm{ICW}$ ratio and $\triangle \mathrm{EI}$, and $\mathrm{C}$ shows the correlation between $\Delta \mathrm{Sum}$-Quad MT and $\triangle \mathrm{ECW} / \mathrm{ICW}$ ratio. The plus symbol $(+)$ indicates the changes at PostEx1 from baseline, the cross $(\times)$ indicates the changes at PostEx2, and the circle $(\circ)$ indicates the changes at PostEx3. 


\section{Figures;}

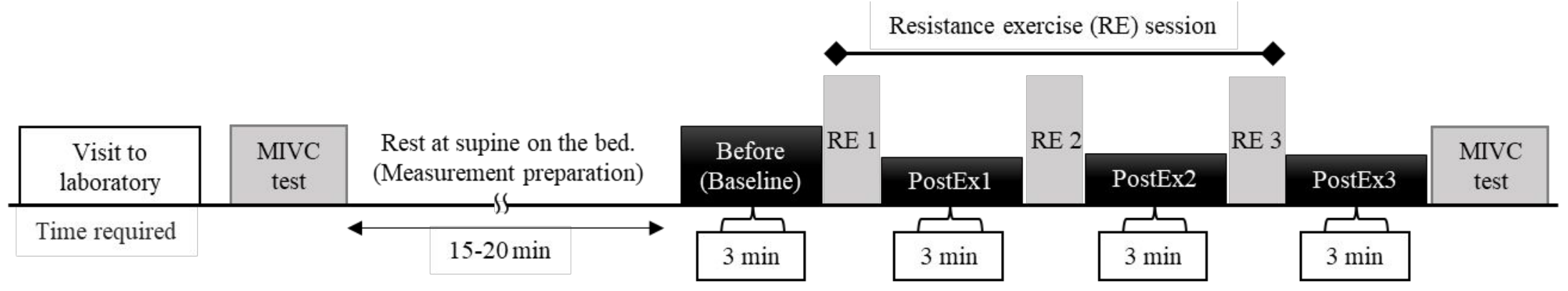

Figure 1 

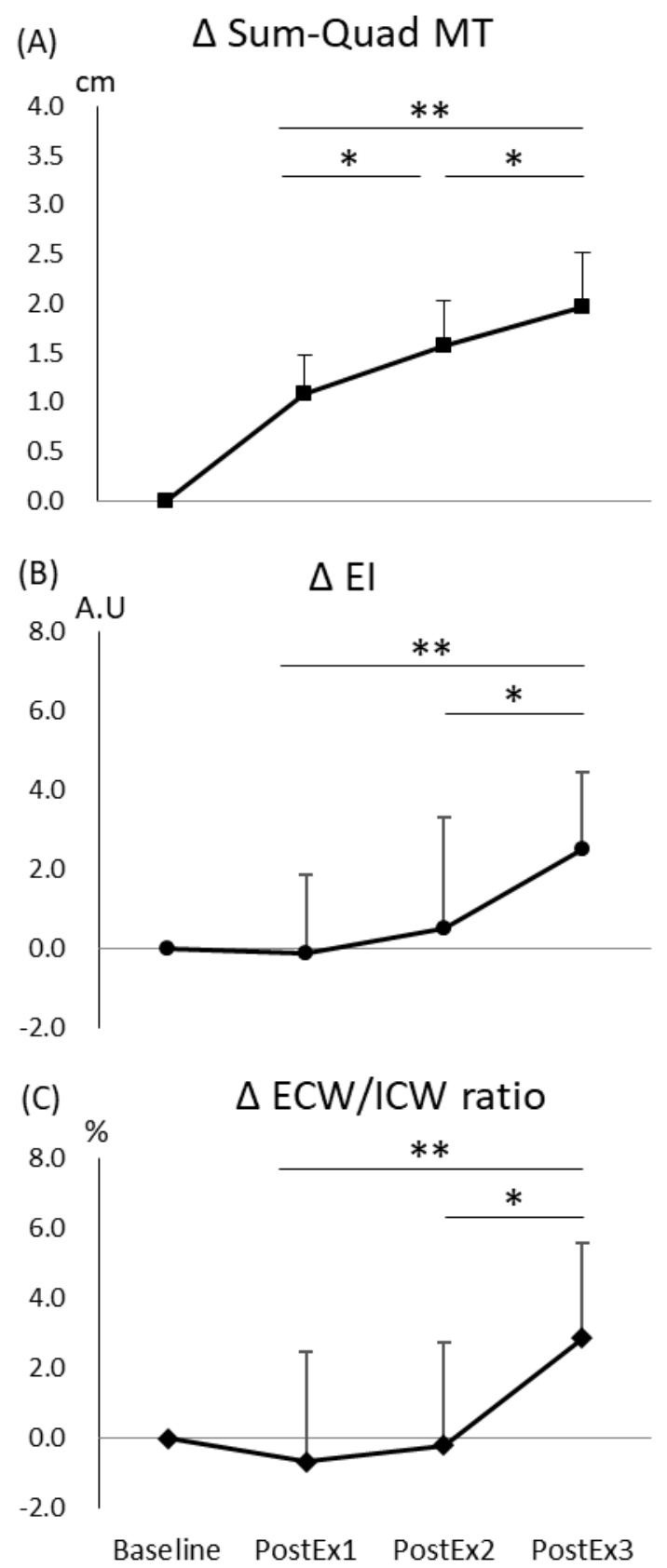

Figure 2 

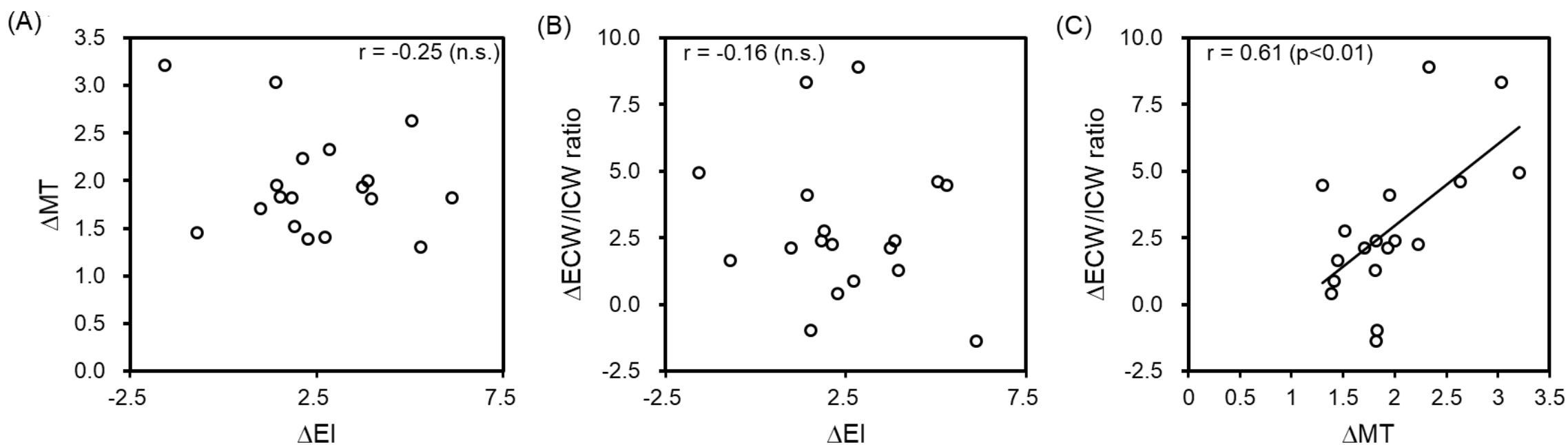

Figure 3 
(A)

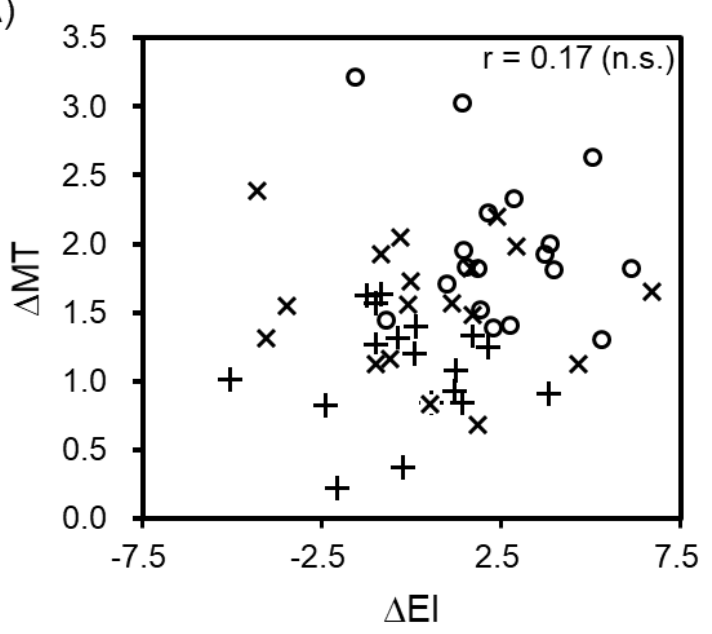

(B)

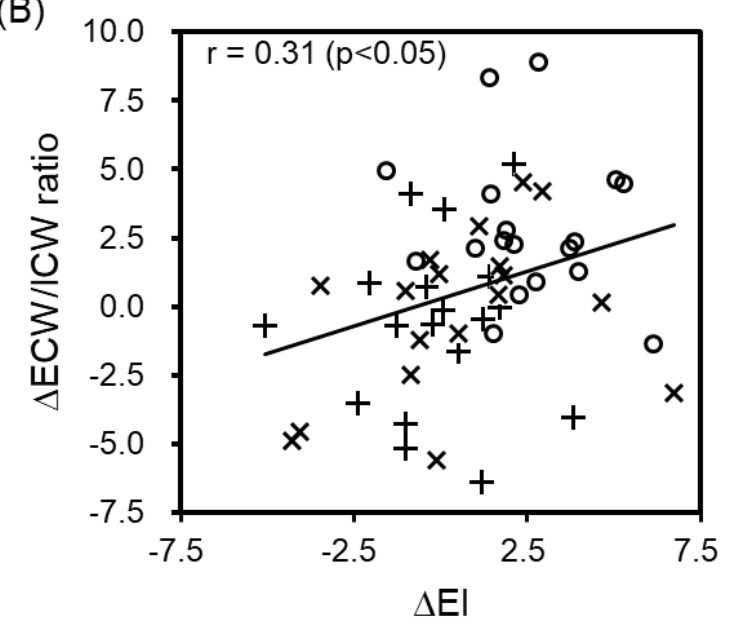

(C)

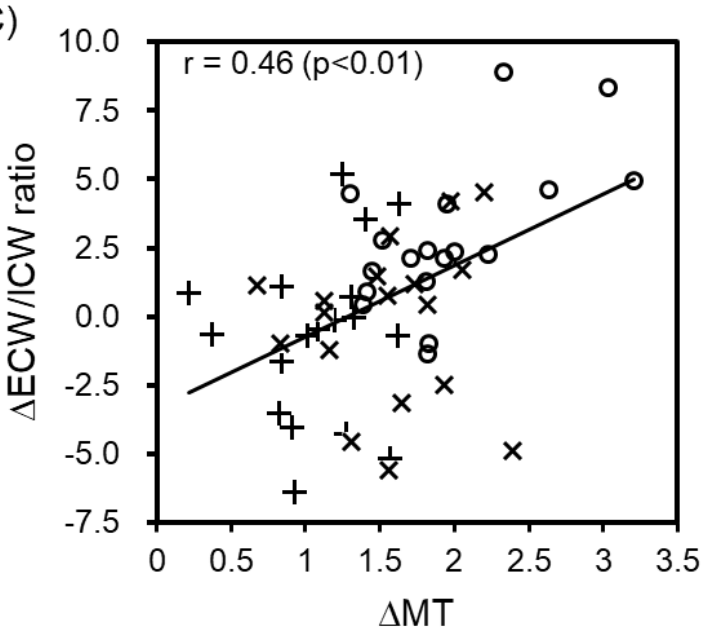

Figure 4 